\title{
Analytic functions concerning with some subordinations
}

\author{
SHIGEYOSHI OWA ${ }^{\dagger}, J_{U N I C H I}$ NISHIWAKI, DANIEL BREAZ AND NICOLETA BREAZ
}

\author{
Date of Receiving : $\quad 01.03 .2017$ \\ Date of Revision : 13.08 .2017 \\ Date of Acceptance : 24.08 .2017
}

\begin{abstract}
For analytic functions in the class $\mathcal{A}_{n}$ in the open unit disk $\mathbb{U}$, two subclasses $\mathcal{S}_{n}^{*}(\alpha)$ and $\mathcal{K}_{n}(\alpha)$ of starlike functions and convex functions are introduced. The object of the present paper is to discuss some interesting properties of functions in the classes $\mathcal{S}_{n}^{*}(\alpha)$ and $\mathcal{K}_{n}(\alpha)$ with some subordinations.
\end{abstract}

\section{Introduction}

Let $\mathcal{A}_{n}$ be the class of functions $f(z)$ of the form

$$
f(z)=z+\sum_{k=n}^{\infty} a_{k} z^{k} \quad(n=2,3,4, \cdots)
$$

which are analytic in the open unit disc $\mathbb{U}=\{z \in \mathbb{C}:|z|<1\}$. If we consider a function $f(z) \in \mathcal{A}_{n}$ which satisfies

$$
\operatorname{Re}\left(\frac{z f^{\prime}(z)}{f(z)}\right)>0 \quad(z \in \mathbb{U})
$$

then we say that $f(z)$ is starlike with respect to the origin in $\mathbb{U}$. We denote the subclass of $\mathcal{A}_{n}$ by $\mathcal{S}_{n}^{*}$ consisting of starlike functions in $\mathbb{U}$. Also, we say that $f(z)$ is convex in $\mathbb{U}$ if $f(z) \in \mathcal{A}_{n}$ satisfies

$$
\operatorname{Re}\left(1+\frac{z f^{\prime \prime}(z)}{f^{\prime}(z)}\right)>0 \quad(z \in \mathbb{U}) .
$$

This is equivalent to $z f^{\prime}(z) \in \mathcal{S}_{n}^{*}$. We denote by $\mathcal{K}_{n}$ the subclass of $\mathcal{A}_{n}$ consisting of all convex functions in $\mathbb{U}$.

Let us consider a function $f(z)$ given by

$$
f(z)=z+\frac{1}{n} z^{n} \quad(n=2,3,4, \cdots) .
$$

2010 Mathematics Subject Classification. 30C45.

Key words and phrases. Analytic function, starlike function, convex function, subordination. Communicated by: Shiv K. Kaushik

${ }^{\dagger}$ Corresponding author. 